\title{
VALIDAÇÃO DA PRECIPITAÇÃO ESTIMADA PELO PRODUTO 3B42 DO SATÉLITE "TROPICAL RAINFALL MEASURING MISSION" PARA A SUB- BACIA HIDROGRÁFICA PARAÍBA DO SUL, ESTADO DE SÃO PAULO, BRASIL
}

\author{
SILVA, Mateus Alexandre da - mateus4lexandre@outlook.com \\ Universidade Federal de Lavras / UFLA \\ MERLO, Marina Neves - marinanevesmerlo@gmail.com \\ Universidade Federal de Lavras / UFLA \\ THEBALDI, Michael Silveira - michael.thebaldi@ufla.br \\ Universidade Federal de Lavras / UFLA \\ VIOLA, Marcelo Ribeiro - marcelo.viola@ufla.br \\ Universidade Federal de Lavras / UFLA
}

Submetido em: $18 / 08 / 2020$

Aceito para publicação em: 05/05/2021

Publicado em: 27/05/2021

DOI: http://dx.doi.org/10.5380/abclima.v28i0.75926

\begin{abstract}
RESUMO: O monitoramento hidrológico em bacias hidrográficas se faz necessário para prevenir e mitigar danos provocados por precipitações, porém, quando este é realizado por meio de observações em campo possui limitações, como: não cobrir todo o território brasileiro, a necessidade de técnicos para operação e estar sujeito à falha de dados em séries históricas. Para suprir a crescente demanda por monitoramento, surgiu o satélite Tropical Rainfall Measuring Mission (TRMM), capaz de monitorar variáveis hidrológicas, como, por exemplo, a precipitação. Objetivou-se com o presente estudo validar os dados de precipitação estimados pelo produto 3B42 do satélite TRMM para a sub-bacia hidrográfica Paraíba do Sul. Foram obtidos dados de precipitação para seis postos no Estado de São Paulo. Os dados estimados pelo satélite TRMM foram obtidos por meio da plataforma EarthData (NASA) e os dados observados por meio da plataforma HIDROWEB (ANA). Após tabulação, procedeu-se a análise de dados diários, mensais e da máxima diária anual por meio dos parâmetros estatísticos de correlação de Pearson, erro médio absoluto, raiz do erro médio ao quadrado, viés e coeficiente de concordância. Os dados diários estimados de precipitação não apresentaram boa correlação com os dados diários observados nos postos. Em contrapartida, os dados mensais obtiveram forte correlação com os dados observados, fato que viabiliza a utilização dos dados fornecidos por satélite em tal escala temporal. Quanto à precipitação máxima diária anual, o satélite não teve precisão satisfatória para realizar tal estimativa.
\end{abstract}

PALAVRAS-CHAVE: Sensoriamento remoto, Hidrologia Aplicada, Atlântico Leste, TRMM, Simulação Hidrológica.

\section{VALIDATION OF PRECIPITATION ESTIMATED BY PRODUCT 3B42 FROM THE SATELLITE "TROPICAL RAINFALL MEASURING MISSION" FOR THE PARAÍBA DO SUL HYDROGRAPHIC SUB-BASIN, STATE OF SÃO PAULO, BRAZIL}

ABSTRACT: Hydrological monitoring in hydrographic basins is necessary to prevent and mitigate damage caused by precipitation, however, when this is done through field observations, it has limitations, such as: not covering the entire Brazilian territory, the need for technicians to operate and be subject to data failure in historical series. To meet the growing demand for monitoring, the Tropical Rainfall Measuring Mission (TRMM) satellite appeared, capable of monitoring hydrological variables, such as, for example, precipitation. The objective of this study was to validate the precipitation data estimated by the product 3B42 of the TRMM satellite for the Paraíba do Sul hydrographic sub-basin. 
Precipitation data were obtained for six stations in the State of São Paulo. The data estimated by the TRMM satellite were obtained using the EarthData platform (NASA) and the observed data from the HIDROWEB platform (ANA). After tabulation, daily, monthly and daily maximum daily data were analyzed using Pearson's correlation statistical parameters, absolute mean error, root of the mean squared error, bias and agreement coefficient. The estimated daily rainfall data did not show a good correlation with the daily data observed at the stations. In contrast, the monthly data obtained a strong correlation with the observed data, a fact that makes it possible to use the data provided by satellite on such a time scale. As for the maximum annual daily precipitation, the satellite did not have satisfactory precision to make such an estimate.

KEYWORDS: Remote sensing, Applied Hydrology, East Atlantic, TRMM, Hydrological Simulation.

\section{VALIDACIÓN DE LA PRECIPITACIÓN ESTIMADA POR EL PRODUCTO $3 B 42$ DEL SATÉLITE "TROPICAL RAINFALL MEASURING MISSION" PARA LA SUBCUENCA HIDROGRÁFICA PARAÍBA DO SUL, ESTADO DE SÃO PAULO, BRASIL}

RESUMEN: El monitoreo hidrológico en las cuencas hidrográficas es necesario para prevenir y mitigar el daño causado por la precipitación, sin embargo, cuando esto se realiza a través de observaciones de campo, tiene limitaciones, tales como: no cubrir todo el territorio brasileño, la necesidad de que los técnicos operen y estén sujeto a falla de datos en series históricas. Para satisfacer la creciente demanda de monitoreo, apareció el satélite de la Tropical Rainfall Measuring Mission (TRMM), capaz de monitorear variables hidrológicas, como, por ejemplo, la precipitación. El objetivo de este estudio fue validar los datos de precipitación estimados por el producto 3B42 del satélite TRMM para la subcuenca hidrográfica de Paraíba do Sul. Se obtuvieron datos de precipitación para seis estaciones en el Estado de São Paulo. Los datos estimados por el satélite TRMM se obtuvieron utilizando la plataforma EarthData (NASA) y los datos observados obtenidos utilizando la plataforma HIDROWEB (ANA). Después de la tabulación, se analizaron los datos diarios máximos diarios, mensuales y diarios utilizando los parámetros estadísticos de correlación de Pearson, error medio absoluto, raíz del error cuadrático medio, sesgo y coeficiente de concordancia. Los datos estimados de lluvia diaria no mostraron una buena correlación con los datos diarios observados en las estaciones. En contraste, los datos mensuales obtuvieron una fuerte correlación con los datos observados, un hecho que hace posible utilizar los datos proporcionados por satélite en tal escala de tiempo. En cuanto a la precipitación diaria máxima anual, el satélite no tuvo una precisión satisfactoria para hacer tal estimación.

PALABRAS CLAVE: Teledetección, Hidrología Aplicada, Atlántico Este, TRMM, Simulación Hidrológica.

\section{INTRODUÇÃO}

As atividades antrópicas, aliadas à falta de consciência e gerenciamento ambiental, continuam a ocasionar a redução da qualidade hídrica, sem qualquer preocupação com as interações ecossistêmicas que possam vir a influenciar o regime hídrico (MELO NETO, 2012). Neste sentido, a bacia hidrográfica do rio Paraíba do Sul, que se estende por três estados do sudeste brasileiro de elevada concentração populacional - São Paulo, Minas Gerais e Rio de Janeiro - possui grande importância não apenas para o abastecimento industrial e doméstico, mas também atua como corpo hídrico receptor de efluentes, podendo, deste modo, afetar sua quantidade e qualidade (ANA, 2015).

Sendo assim, o monitoramento hidrológico vem avançando rumo ao conhecimento da distribuição dos eventos de chuva e de seca nas bacias hidrográficas, os quais possuem importância hidrológica, ecológica e socioeconômica. (CARVALHO; SHOEGIMA; NERY, 2017). Além disso, visa 
atender às necessidades de redução dos impactos gerados pela ocupação desordenada das cidades, como o escoamento superficial direto da água de chuva, drenagem urbana e abastecimento público (FIGUEIREDO et al., 2010; GARCIA; PAIVA, 2006). Contudo, a rede de monitoramento traz certa carência de dados hidrológicos observados em campo, gerando lacunas no espaço e no tempo em relação à disponibilidade destes (MELO NETO, 2012).

Tornou-se, então, crescente a utilização do sensoriamento remoto para suprir às necessidades de informação sobre a precipitação pluvial, desde a década de 90. Um dos radares utilizados para tal finalidade é o Tropical Rainfall Measuring Mission (TRMM), projetado especificamente para monitorar e estimar a chuva nos trópicos, oferecendo informações minuciosas sobre a estrutura da mesma (KUMMEROW et al., 2000). Os dados possuem resolução temporal e espacial considerados altos, já que o satélite possui órbita oblíqua, o que promove um período de translação curto com maior amostragem de dados, cuja resolução é de aproximadamente $25 \times 25 \mathrm{~km}$ (NÓBREGA; DE SOUZA; GALVÍNCIO, 2008; SILVA; PANTANO; CAMARGO, 2013).

Segundo Fensterseifer, Allasia e Paz (2016), o produto 3B42 versão 7 do TRMM possui a capacidade de estimar a precipitação pluvial com uma resolução espacial de $0,25^{\circ} \times 0,25^{\circ}$ e temporal de três horas. Almeida et al. (2020) salientam ainda este produto usa duas bandas espectrais - TRMM Microwave Imager (TMI) e Precipitation Radar (PR) - para a estimativa da precipitação.

As informações geradas pelo produto 3B42 advindos do sensoriamento remoto são instrumentos importantes para a obtenção de dados, assim como um auxílio para preenchimento de falhas de precipitação (PESSI et al., 2019). Ainda que sejam necessárias validações dos produtos gerados pelo satélite por meio de observações confiáveis em superfície, a fim de avaliar a precisão dos dados TRMM (ANJOS; CANDEIAS; NÓBREGA, 2016; ARAÚJO; SANCHES; FERREIRA, 2017; COLLISCHONN, 2006; COLLISCHONN et al., 2007; DA SILVA; PRELA-PANTANO CAMARGO, 2013; PESSI et al., 2019; KARASEVA; PRAKASH; GAIROLA, 2011; KUMMEROW et al., 2000), as estimativas trazem informações geograficamente espacializadas sobre a distribuição das chuvas, além de serem um meio de monitoramento e gestão dos recursos hídricos (COLLISCHONN, 2006; MACEDO, 2013).

Desta forma, objetivou-se com este trabalho validar os dados obtidos pelo produto 3B42 do satélite TRMM, tendo como base os dados observados em superfície nos postos pluviométricos para a sub-bacia Paraíba do Sul, na bacia hidrográfica Atlântico Leste.

\section{MATERIAL E MÉTODOS}

Foram selecionados 6 postos pluviométricos situados no estado de São Paulo, dentro da sub-bacia Paraíba do Sul, da bacia hidrográfica Atlântico Leste. As coordenadas geográficas do quadrante, no qual os postos estão incluídos, são $29,159^{\circ} \mathrm{S}$ e $20,106^{\circ} \mathrm{S}$ de latitude e $51,108^{\circ} \mathrm{W}$ e $38,979^{\circ} \mathrm{W}$ de longitude.

O clima da região é classificado segundo Köppen como Cwa - tropical de altitude, com inverno seco e chuvas de verão, Cfa - subtropical úmido, sem estação seca e com verão quente, e Cfb - subtropical úmido, sem estação seca 
e com verão temperado (ALVARES, 2013). A localização dos postos é identificada na Figura 1.

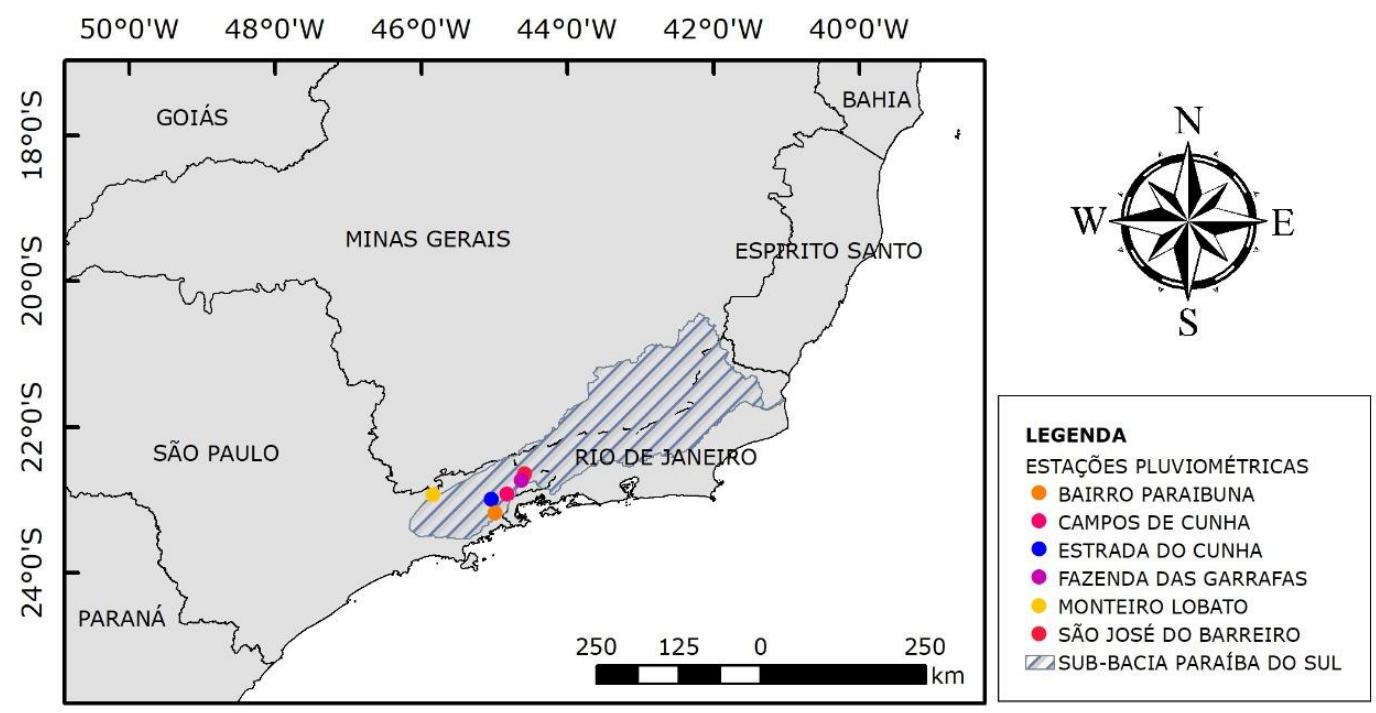

Figura 1 - Localização dos postos pluviométricos da bacia hidrográfica Atlântico Leste, situada na porção oeste do estado de São Paulo. Fonte: Os autores, adaptado de ANA (2019).

Para a realização do estudo foi definido um período de 19 anos, compreendidos entre 1998 e 2016, utilizando a metodologia de comparação ponto-a-ponto, assim como utilizado por Soares, Paz e Piccili (2016). Os dados observados nos postos pluviométricos foram obtidos por meio da base de dados disponibilizada no site HIDROWEB, da Agência Nacional de Águas (ANA, 2019).

As séries de precipitação total mensal foram obtidas pela soma dos dados diários observados, preenchendo-se as falhas destes, quando verificadas, pelo método Inverso do Quadrado da Distância, conforme apresentado por Mello e Silva (2013).

Com isso, foram determinadas as precipitações totais anuais de cada posto no intervalo de tempo estudado, verificada a homogeneidade da região onde os postos estão inseridos bem como a consistência dos dados por meio da Curva de Dupla Massa (ANA, 2012). Esta curva foi obtida tendo como referência o posto pluviométrico no qual os dados disponibilizados eram consistidos, que neste caso foi o posto Estrada do Cunha.

Os dados de precipitação diária do produto 3B42 do satélite TRMM (versão 7), por outro lado, disponíveis no site EarthData (NASA), foram obtidos pelas coordenadas geográficas de cada um dos postos e convertidos para o formato .xIs utilizando o software RStudio. Desta forma, os dados passaram por tabulação, obtendo-se a precipitação mensal e a precipitação máxima diária anual. A obtenção da precipitação máxima diária anual se deu observando-as nas séries históricas diárias de precipitação de cada um dos postos pluviométricos. Para sua validação, estas foram comparadas com a precipitação diária ocorrida na mesma data estimada pelo satélite TRMM. 
A validação dos dados de precipitação diária, mensal e máxima diária anual do produto 3B42 do satélite TRMM foram feitas por meio do cálculo do coeficiente de correlação linear de Pearson $(r)$ erro médio absoluto (MAE, em $\mathrm{mm}$ ), raiz quadrada do erro médio quadrático (RMSE, em $\mathrm{mm}$ ) e a média do erro sistemático (Viés, em $\mathrm{mm}$ ), os quais estão representados pelas Equações 1 , 2,3 e 4, respectivamente.

$$
\begin{gathered}
r=\frac{\sum_{\mathrm{i}=1}^{\mathrm{n}}\left(\mathrm{Est}_{\mathrm{i}}-\overline{\mathrm{Est}}\right) *\left(\mathrm{Obs}_{\mathrm{i}}-\overline{\mathrm{Obs}}\right)}{\sqrt{\left[\sum_{\mathrm{i}=1}^{\mathrm{n}}(\mathrm{Est}-\overline{\mathrm{Est}})^{2}\right] *\left[\sum_{\mathrm{i}=1}^{\mathrm{n}}(\mathrm{Obs}-\overline{\mathrm{Obs}})^{2}\right]}} \\
\text { MAE }=\frac{1}{\mathrm{~N}} * \sum_{\mathrm{i}=1}^{\mathrm{n}}(|\mathrm{Est}-\mathrm{Obs}|) \\
\operatorname{RMSE}=\left[\frac{1}{\mathrm{~N}} * \sum_{\mathrm{i}=1}^{\mathrm{n}}\left(\mathrm{Est}_{\mathrm{i}}-\mathrm{Obs}\right)^{2}\right]^{\frac{1}{2}} \\
\text { Viés }=\frac{1}{\mathrm{~N}} * \sum_{\mathrm{i}=1}^{\mathrm{N}}\left(\text { Est }_{\mathrm{i}}-\mathrm{Obs}_{\mathrm{i}}\right)
\end{gathered}
$$

Em que, "Est" representa os dados estimados pelo TRMM, "Obs" os dados observados nos postos pluviométricos de superfície, " $\overline{\mathrm{Est}}$ " e " $\overline{\mathrm{Obs}}$ " são as médias dos dados TRMM e postos pluviométricos, consecutivamente, e $N$ é a quantidade de dados de cada uma das validações.

Além disso, foram calculados o coeficiente de determinação $\left(R^{2}\right)$, que indica o quanto a variável dependente (produtos 3B42) foi explicada pela variável independente (postos pluviométricos) (WILLMOTT; ACKLESON; DAVIS, 1985), e o coeficiente de concordância - d (Equação 5), que aponta o grau de exatidão entre os valores estimados e observados, no sentido da maior aproximação de 1, indicado pela reta 1:1 (SILVA; PANTANO; CAMARGO, 2013).

$$
\mathrm{d}=1-\left[\frac{\sum_{\mathrm{i}=1}^{\mathrm{n}}\left(\mathrm{Est}_{\mathrm{i}}-\mathrm{Obs}_{\mathrm{i}}\right)^{2}}{\sum_{\mathrm{i}=1}^{\mathrm{n}}\left(\left|\mathrm{Est}_{\mathrm{i}}-\overline{\mathrm{Obs}}\right|+\left|\mathrm{Obs}_{\mathrm{i}}-\overline{\mathrm{Obs}}\right|\right)^{2}}\right]
$$

Para estabelecer uma comparação da variabilidade do RMSE para cada posto pluviométrico em diferentes escalas temporais calculou-se também o coeficiente de variação ( $\left.\mathrm{CV}_{\mathrm{RMSE}}\right)$ - Equação 6.

$$
\mathrm{CV}_{\mathrm{RMSE}}=\frac{\mathrm{RMSE}}{\overline{\mathrm{ObS}}}
$$

\section{RESULTADOS E DISCUSSÃO}

VERIFICAÇÃO DA HOMOGENEIDADE DO LOCAL DE ESTUDO E CONSISTÊNCIA DOS DADOS

O gráfico da precipitação anual acumulada dos postos a serem avaliados em função da precipitação acumulada do posto de referência está apresentado na Figura 2. 


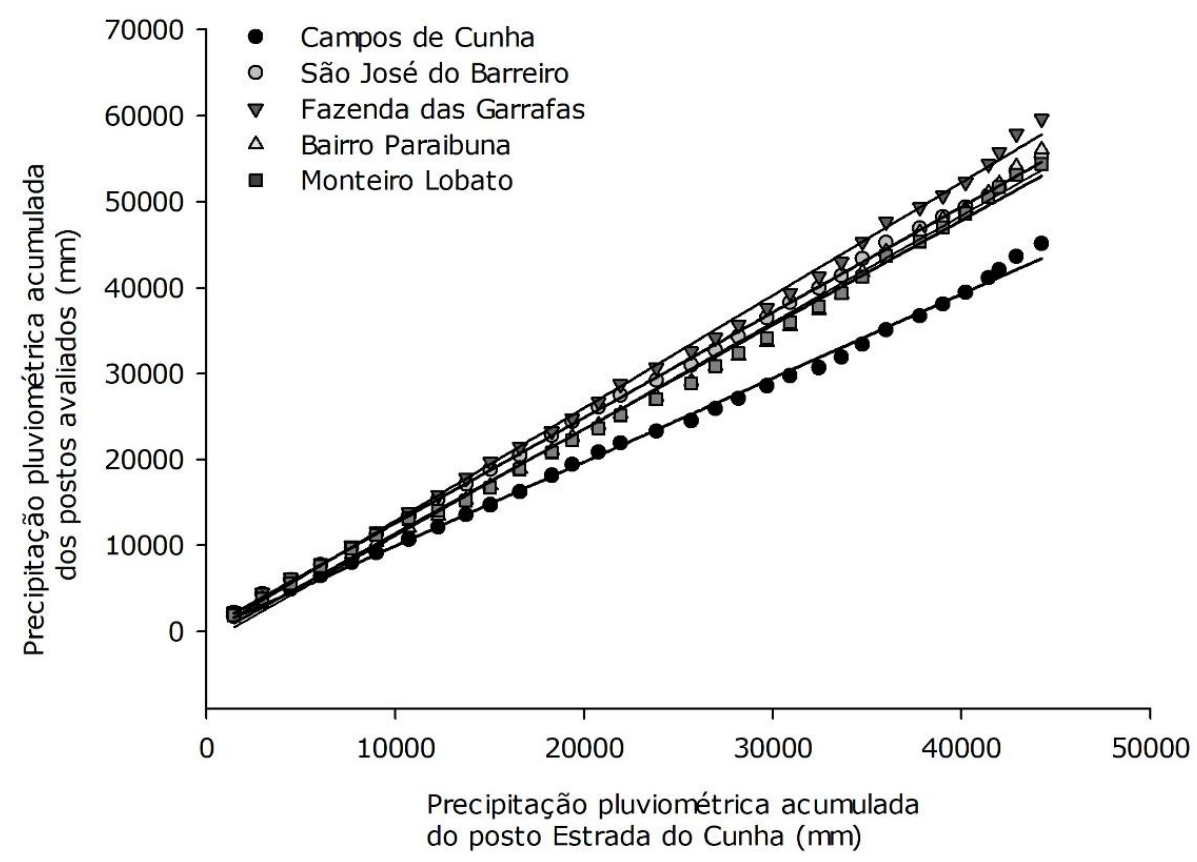

Figura 2 - Análise de consistência dos postos pluviométricos contidos na sub-bacia Paraíba do Sul, pelo método da Curva de Dupla Massa, e suas respectivas linhas de tendência (linha sólidas). Fonte: Os autores (2020).

A análise de consistência dos dados dos cinco postos pluviométricos avaliados em relação ao posto de referência (Estrada do Cunha), foi realizada por meio de ajuste de linha de tendência linear, cujo coeficiente de determinação, em média, foi de $99,73 \%$ (Figura 2). Assim, pode-se eliminar possíveis erros de transcrição dos dados observados em campo ou alteração do coeficiente angular da reta.

\section{VALIDAÇÃO DOS DADOS DE PRECIPITAÇÃO DIÁRIA}

A validação dos produtos TRMM ocorreu por meio da análise dos 6 postos pluviométricos, considerando-se, inicialmente, os dados diários do período de 1998 a 2016, os quais podem ser visualizados na Figura 3 (A, B, C, D, E e F). 

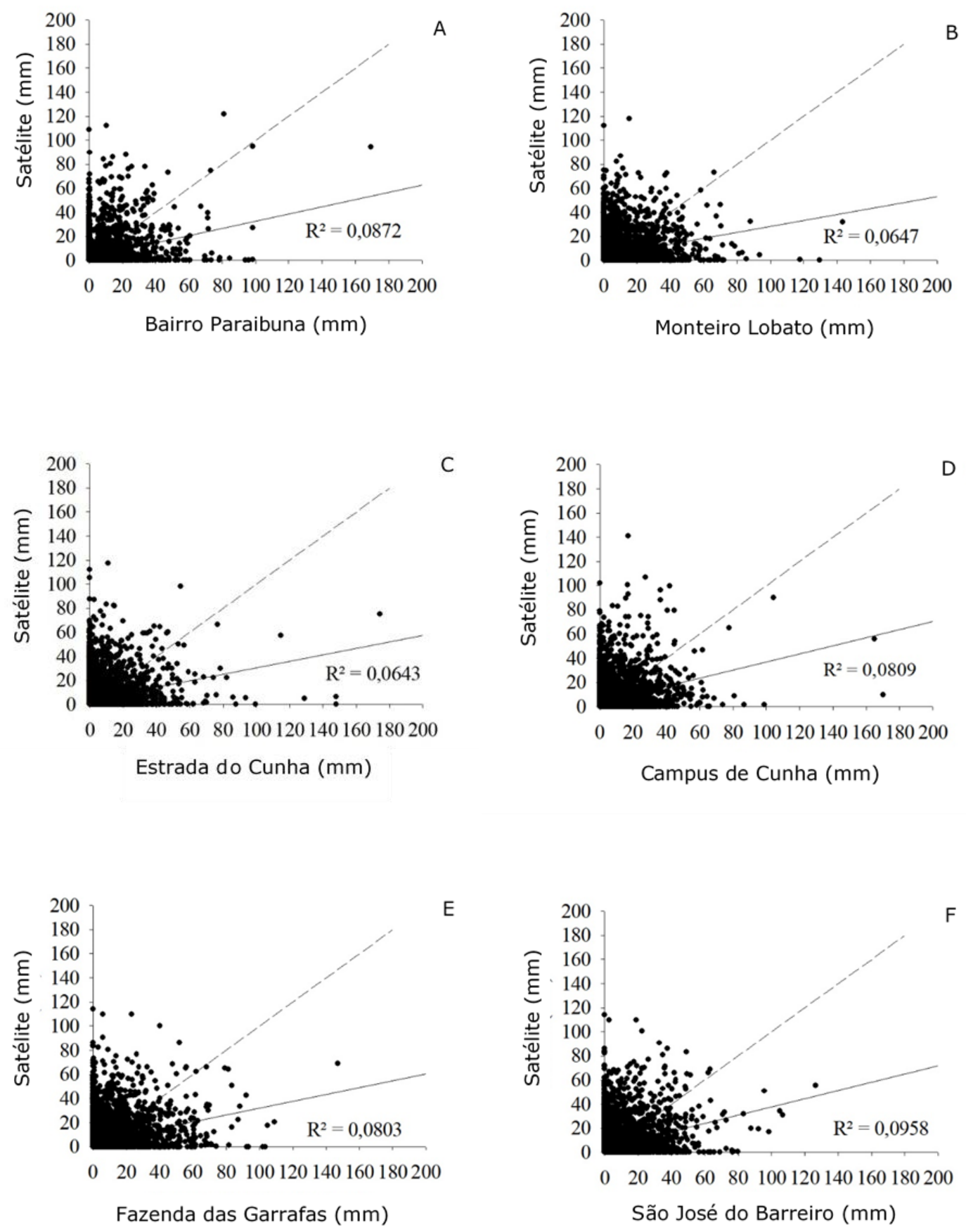

Figura 3 - Relação entre os dados diários de precipitação estimados pelo produto 3B42 do satélite TRMM e os dados observados nos postos pluviométricos da sub-bacia Paraíba do Sul, no período de 1998 a 2016; linha de tendência (linha sólida) e linha de proporção 1:1 (linha tracejada). Fonte: Os autores (2020). 
É possível notar que não se obteve bons coeficientes de determinação relacionando os dados diários observados e estimados, estando compreendidos entre 6,43 e 9,58\% de adequação entre estes. Além disso, a linha de tendência dos dados não se aproximou da reta $1: 1$, indicando baixa correlação. Os outros indicadores estatísticos para a precipitação diária estão apresentados na Tabela 1 .

Tabela 1 - Coeficiente de correlação linear de Pearson, erro médio absoluto, raiz quadrada do erro médio quadrático, viés, coeficiente de concordância e $\mathrm{CV}_{\text {RMSE, }}$ calculados por meio dos dados diários observados nos postos pluviométricos e os dados estimados pelo produto 3B42 do satélite TRMM, no período de 1998 a 2016.

\begin{tabular}{ccccccc} 
Postos pluviométricos & $r$ & MAE $(\mathrm{mm})$ & $\mathrm{RMSE}(\mathrm{mm})$ & Viés $(\mathrm{mm})$ & $\mathrm{d}$ & $\mathrm{CV}_{\mathrm{RMSE}}$ \\
\hline Bairro Paraibuna & 0,30 & 6,08 & 12,39 & $-0,94$ & 0,54 & 2,45 \\
Monteiro Lobato & 0,25 & 5,95 & 12,42 & $-0,09$ & 0,50 & 2,65 \\
Estrada do Cunha & 0,25 & 5,48 & 12,19 & 0,94 & 0,48 & 3,40 \\
Campos de Cunha & 0,28 & 5,53 & 12,04 & 0,97 & 0,50 & 3,25 \\
Fazenda das Garrafas & 0,28 & 6,40 & 13,23 & $-0,20$ & 0,52 & 2,60 \\
São José do Barreiro & 0,31 & 5,96 & 12,49 & 0,48 & 0,54 & 2,81 \\
\hline
\end{tabular}

Fonte: Os autores (2019).

As correlações de Pearson encontradas para os dados diários dos postos pluviométricos estudados em relação aos dados TRMM, cujos coeficientes ficaram entre 0,25 e 0,31, foram fracas, segundo Dancey e Reidy (2005). Os mesmos autores classificam os coeficientes de Pearson como: fraco, para $r=$ 0,10 a 0,30, moderado, para $r=0,40$ a 0,60, e forte, para $r=0,70$ a 1,00. Assim, quanto mais próximo a 1,00, maior o grau estatístico de dependência linear entre as variáveis (FIGUEIREDO FILHO; SILVA JÚNIOR, 2009).

A baixa correlação entre os dados pode estar relacionada à análise ponto a ponto, onde um ponto qualquer pode não representar a precipitação da área de abrangência de um pixel TRMM. Para mais, as estimativas do TRMM são feitas a cada 3 horas (FERREIRA; VARONE; ALVES, 2012), na qual a precipitação estimada é a média de cada pixel, cuja área de abrangência é de $625 \mathrm{~km}^{2}$ (PEREIRA et al, 2013).

Os autores Soares, Paz e Piccilli (2016), em contrapartida, para um estudo realizado para mesorregiões no estado da Paraíba, obtiveram um maior coeficiente de correlação entre os valores diários estimados e observados, para os quais as linhas de tendência se aproximaram da reta 1:1. Os valores diários deste índice encontrados pelos autores variaram entre 0,75 e 0,89, entretanto, foram utilizados 269 postos para a caracterização das mesorregiões, em um período de 14 anos, diferentemente do avaliado neste estudo, em que buscouse verificar a precisão dos produtos gerados pelo TRMM pontualmente, em relação a postos pluviométricos de superfície.

O erro médio absoluto da precipitação diária variou entre 5,48 e 6,40 $\mathrm{mm}$. De maneira semelhante, para a raiz do erro médio quadrático, obteve-se para os postos valores similares, compreendidos entre 12 e $13 \mathrm{~mm}$. Nestes casos, denota-se que houve uma variação de até $25 \%$ entre os coeficientes de variação $\left(\mathrm{CV}_{\mathrm{RMSE}}\right)$. No entanto, apesar da média do RMSE não variar entre as estações, pode-se verificar por meio do $\mathrm{CV}_{\text {RMSE }}$ que houve uma grande amplitude dos referidos erros em cada estação. 
O Viés apresentou valores baixos, que em geral, denotaram tanto subestimativa $(-0,94 \mathrm{~mm})$ quanto superestimativa $(0,97 \mathrm{~mm})$ de precipitação do modelo TRMM em relação aos dados observados. Assim, metade dos postos apresentaram tendência a superestimar os dados e a outra metade, subestimou. O posto pluviométrico Monteiro Lobato foi o que apresentou maior aproximação ao dado observado, com viés de $-0,09 \mathrm{~mm}$, seguido do posto Fazenda das Garrafas $(-0,20 \mathrm{~mm})$. Para o coeficiente de concordância, entretanto, os valores encontrados ficaram compreendidos em torno de $50 \%$ para todos os postos analisados.

Já no trabalho realizado por Viana, Ferreira e Conforte (2010) para a região Sul do Brasil, no período de 1998 a 2007, o Viés mostrou que, em média, houve superestimativa de $0,33 \mathrm{~mm}$ dos dados estimados. Além disso, foram encontrados RMSE de 7,8 a 14,7 mm por dia. Entretanto, as correlações de Pearson para os dados dos autores (média de 0,68 ) foram maiores do que os encontrados neste estudo. Estes utilizaram 10 locais amostrais que continham de três a cinco postos pluviométricos em cada pixel, visando uma melhor estimativa do total precipitado registrado pelo satélite. Fato este que pode ser justificado devido ao posto pluviométrico ser capaz de cobrir uma área de apenas $1 \mathrm{~m}^{2}$ (KIDDER; HAAR, 1995).

\section{VALIDAÇÃO DOS DADOS DE PRECIPITAÇÃO MENSAL}

A correlação dos dados mensais estimados pelo satélite TRMM dos 6 postos estudados, no período de 1998 a 2016, podem ser avaliados pela Figura 4 ( $A, B, C, D, E$ e $F)$.
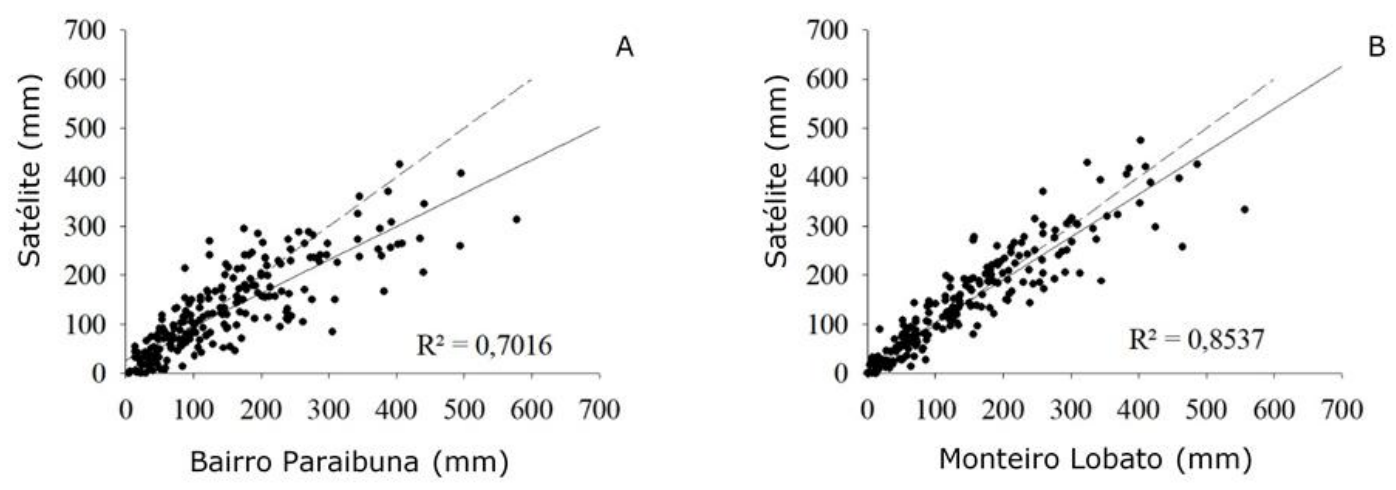

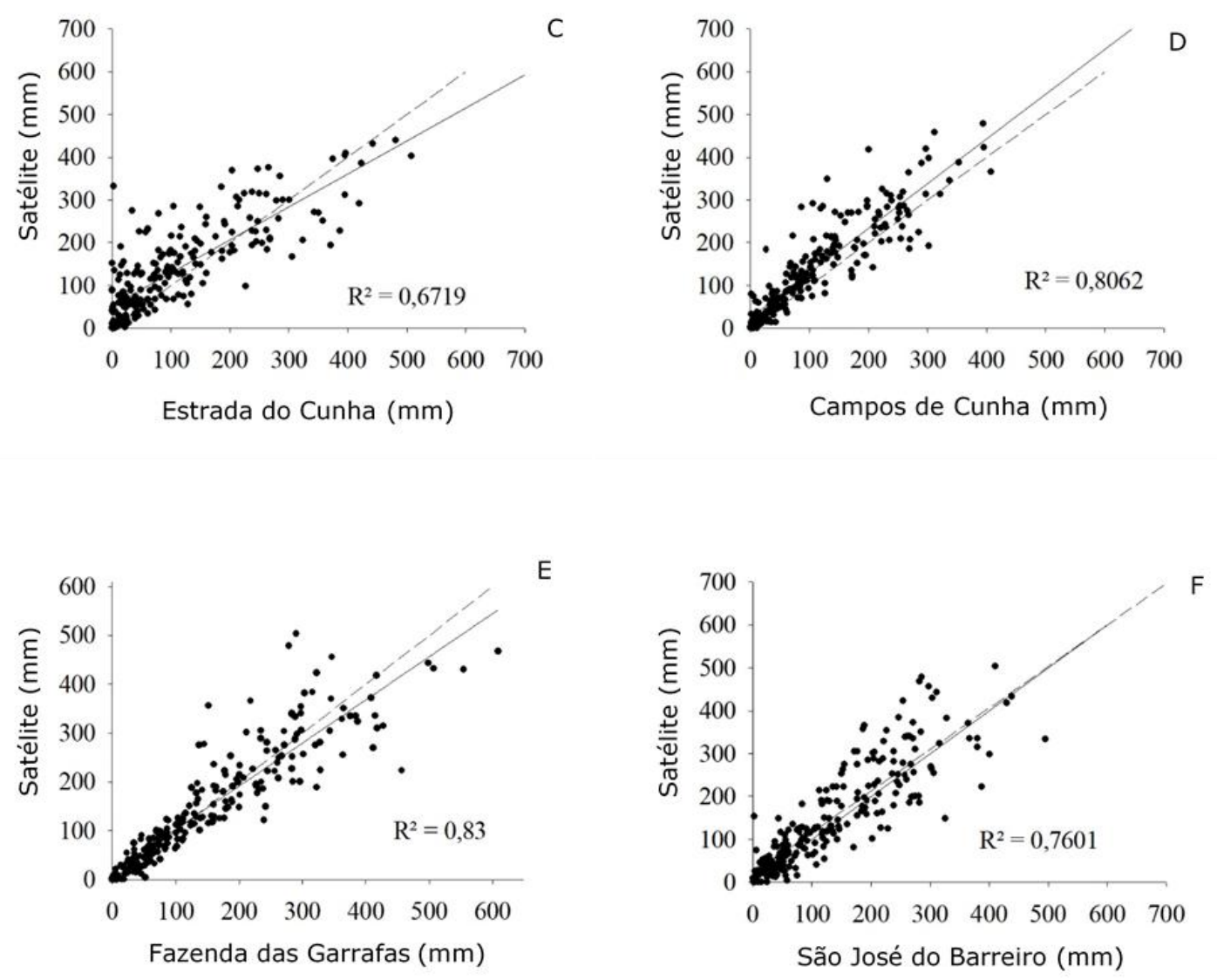

Figura 4 - Relação entre os dados mensais de precipitação estimados pelo produto 3B42 do satélite TRMM e os dados observados nos postos pluviométricos da sub-bacia Paraíba do Sul, no período de 1998 a 2016; linha de tendência (linha sólida) e linha de proporção 1:1 (linha tracejada). Fonte: Os autores (2019).

Por meio da análise da Figura 4, nota-se que os dados mensais observados e estimados obtiveram coeficientes de determinação considerados fortes, os quais ficaram compreendidos entre 67,19 e 85,37\% de adequação entre os dados, sendo que apenas o posto Estrada do Cunha teve seu $\mathrm{R}^{2}$ menor que $70 \%$. Observa-se também que a linha de tendência dos dados se assemelhou a da reta $1: 1$, indicando boa correlação entre os dados observados e estimados. Os demais indicadores estatísticos para a precipitação mensal são mostrados na Tabela 2. 
Tabela 2 - Coeficiente de correlação linear de Pearson, erro médio absoluto, raiz quadrada do erro médio quadrático, viés, coeficiente de concordância e CV $_{\text {RMSE, }}$ calculados por meio dos dados mensais observados nos postos pluviométricos e os dados estimados pelo produto 3B42 do satélite TRMM, no período de 1998 a 2016.

\begin{tabular}{ccccccc} 
Postos pluviométricos & $r$ & MAE $(\mathrm{mm})$ & RMSE $(\mathrm{mm})$ & Viés $(\mathrm{mm})$ & $\mathrm{d}$ & $\mathrm{CV}_{\mathrm{RMSE}}$ \\
\hline Bairro Paraibuna & 0,84 & 44,05 & 64,57 & $-19,03$ & 0,90 & 0,44 \\
Monteiro Lobato & 0,92 & 29,06 & 43,33 & $-0,94$ & 0,96 & 0,31 \\
Estrada do Cunha & 0,82 & 50,67 & 71,14 & 28,32 & 0,89 & 0,65 \\
Campos de Cunha & 0,90 & 39,00 & 55,95 & 29,31 & 0,92 & 0,50 \\
Fazenda das Garrafas & 0,91 & 33,39 & 51,65 & $-3,14$ & 0,95 & 0,34 \\
São José do Barreiro & 0,87 & 42,74 & 60,68 & 14,70 & 0,93 & 0,45 \\
\hline
\end{tabular}

Fonte: Os autores (2019).

As correlações de Pearson obtidas para os dados mensais observados e estimados, segundo Dancey e Reidy (2005), foram fortes, apresentando alto grau estatístico de dependência entre as variáveis $(0,82$ a 0,92).

Comparando as correlações obtidas para os dados diários e mensais, observa-se que os dados mensais apresentaram maior coeficiente de Pearson. Soares, Paz e Piccilli (2016) evidenciaram que quanto maior o período temporal de acumulação pluvial, melhores são as correlações entre os dados observados nos postos pluviométricos e estimados pelo TRMM. Além disso, os mesmos autores completam que isto permite uma compensação dos erros temporais dos dados estimados, em outras palavras, a análise mensal apresenta menor variação entre os resultados quando comparado com dados diários.

Para o erro médio absoluto, foram obtidos valores compreendidos entre 29,06 e 50,67 mm e para a raiz do erro médio quadrático, 43,33 e 71,14 mm. Analisando o $\mathrm{CV}_{\mathrm{RMSE}}$, pode-se observar que houve uma variação superior a $50 \%$ entre os valores de RMSE. Nota-se ainda que, apesar da amplitude do RMSE ser inferior à escala diária, estes valores ainda são elevados.

Tal diferença pode ser explicada pela maior área de cobertura de um pixel TRMM em relação a área de cobertura de um pluviômetro, como supracitado. Pessi et al. (2018) encontraram o erro médio absoluto entre 4,47 e $264,62 \mathrm{~mm}$, em que os maiores valores ocorreram nos meses de alta pluviosidade. Assim, valores elevados para o erro médio absoluto podem ser associados às regiões cujo índice pluviométrico é alto, como no estudo apresentado realizado na região Amazônica.

Para auxiliar na análise do viés e do coeficiente de concordância, a variação temporal da adequação dos dados acumulados mensais observados em relação aos simulados são mostrados na Figura 5. 

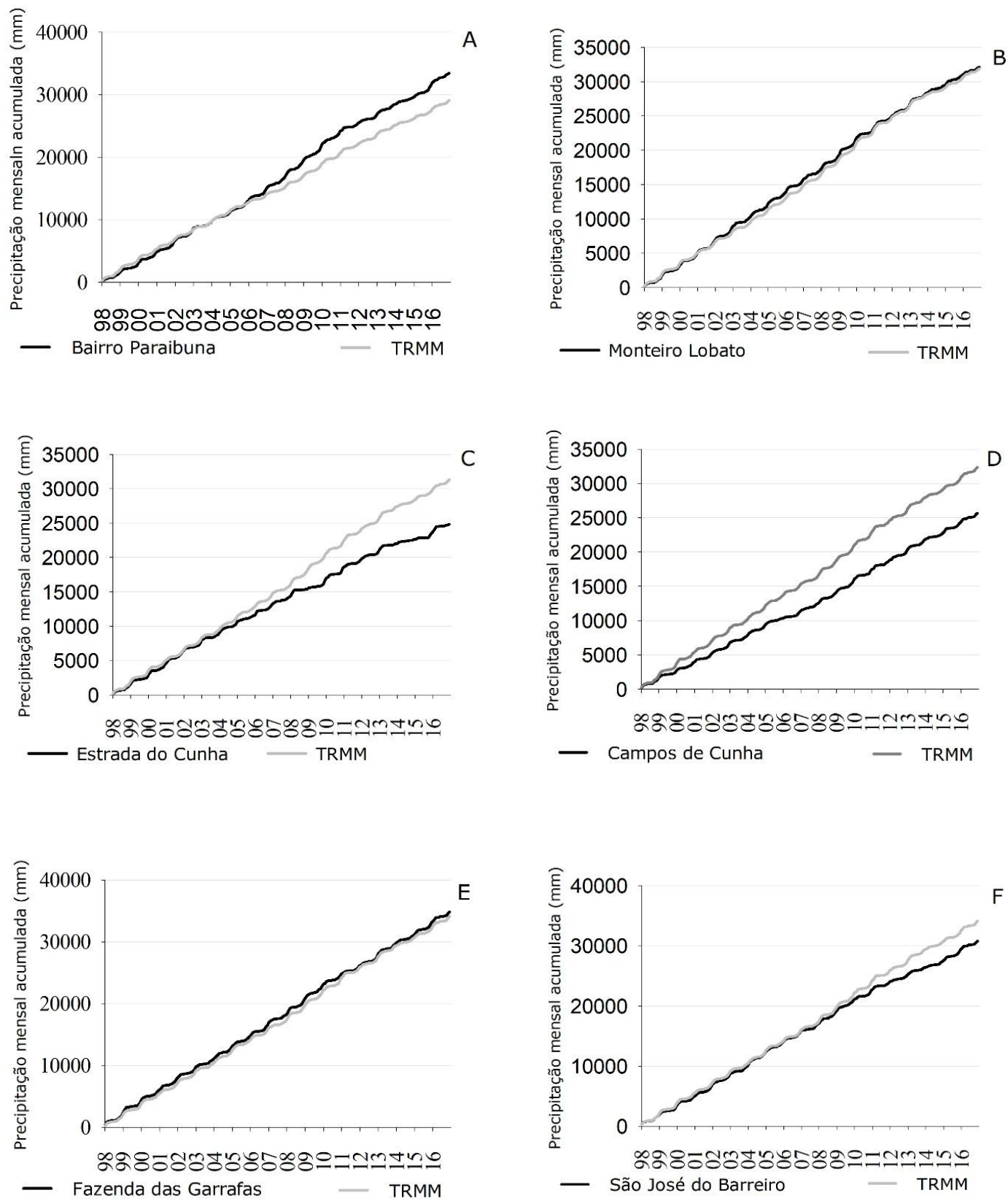

Figura 5 - Variação temporal entre a precipitação acumulada mensal para os postos pluviométricos da sub-bacia Paraíba do Sul e os dados estimados pelo produto 3B42 do satélite TRMM, no período de 1998 a 2016. Fonte: Os autores (2020).

Por meio da análise do viés e dos gráficos da Figura 5, observa-se que os dados não apresentaram generalização na tendência de sub ou superestimativa. Os dados do posto Monteiro Lobato, apesar de terem sido subestimados $(-0,94$ 
$\mathrm{mm}$ ), foram os que mais se aproximaram dos dados observados, como pode ser visto pela sobreposição das linhas na Figura 5B.

Este mesmo posto apresentou também menores erro médio absoluto $(29,06 \mathrm{~mm})$ e raiz do erro médio quadrático $(43,33 \mathrm{~mm})$, e maiores correlação de Pearson $(0,92)$ e coeficiente de concordância $(96 \%)$. Isto se dá devido à maior proximidade entre os dados estimados pelo satélite e os observados em campo.

Pela análise dos gráficos da Figura 5 e pelos resultados dos coeficientes de concordância (Tabela 2), pode-se dizer que foram obtidas relações satisfatórias, as quais estão compreendidas entre 0,89 e 0,96. Tal resultado corrobora com o obtido na correlação de Pearson, em que todos os postos apresentaram forte relação entre os dados observados e simulados.

Para mais, pode-se inferir que a estimativa do TRMM subestimou a precipitação na área próxima ao litoral e superestimou nas áreas mais afastadas. Este fato também foi relatado por Soares, Paz e Piccilli (2016) onde foi subestimado as áreas com maiores índices pluviométricos e superestimado as áreas com menores índices pluviométricos.

\section{VALIDAÇÃO DOS DADOS DE PRECIPITAÇÃO MÁXIMA DIÁRIA ANUAL}

Com relação aos dados de precipitação máxima diária anual, os indicadores estatísticos são apresentados na Tabela 3.

Tabela 3 - Coeficiente de correlação linear de Pearson, erro médio absoluto, raiz quadrada do erro médio quadrático, viés, coeficiente de concordância e CV $_{\text {RMSE }}$ calculados por meio dos dados de precipitação máxima diária anual observados nos postos pluviométricos e os estimados pelo produto 3B42 do satélite TRMM, no período de 1998 a 2016.

\begin{tabular}{cccccccc} 
Postos pluviométricos & $r$ & MAE $(\mathrm{mm})$ & $\mathrm{RMSE}(\mathrm{mm})$ & Viés $(\mathrm{mm})$ & $\mathrm{d}$ & $\mathrm{CV}_{\mathrm{RMSE}}$ \\
\hline Bairro Paraibuna & 0,47 & 23,17 & 29,74 & 1,91 & 0,45 & 0,37 \\
Monteiro Lobato & 0,42 & 17,31 & 26,48 & $-4,83$ & 0,43 & 0,35 \\
Estrada do Cunha & 0,34 & 25,90 & 36,14 & $-11,12$ & 0,27 & 0,44 \\
Campos de Cunha & 0,71 & 23,43 & 27,99 & 7,71 & 0,45 & 0,38 \\
Fazenda das Garrafas & $-0,25$ & 26,27 & 31,71 & $-1,70$ & 0,21 & 0,38 \\
São José do Barreiro & 0,27 & 25,84 & 31,30 & 17,17 & 0,59 & 0,48 \\
\hline
\end{tabular}

Fonte: Os autores (2020).

As correlações de Pearson entre os dados observados e estimados para os postos variaram de 0,27 a 0,71 para cinco dentre os seis postos estudados. 0 posto Fazenda das Garrafas, entretanto, apresentou correlação inversa, indicando que os dados observados e estimados vão em direção oposta. As baixas correlações e a correlação inversa podem estar relacionadas à eventos de alta intensidade e curta duração que ocorrem de forma pontual (CAMPAROTTO et al., 2013). Neste sentido, a informação presente no pixel do TRMM é a média para sua área $\left(625 \mathrm{~km}^{2}\right)$, distorcendo assim informações pontuais.

Para os dados de máxima diária anual, os erros médios absolutos e as raízes dos erros médios quadráticos variaram entre 17,31 e 26,27 mm e 26,48 e $36,14 \mathrm{~mm}$, respectivamente. Apesar dos elevados valores dos erros absolutos, 
teve-se, em média, uma variação inferior do RMSE em cada estação quando comparada à escala mensal, o que pode ser visto pelos valores de CV $\mathrm{CV}_{\text {RME }}$. Notase ainda uma menor variação entre os $\mathrm{CV}_{\mathrm{RMSE}}$ das estações.

Por outro lado, valores de viés que denotam subestimativa de 11,12 mm até valores de superestimativa de $17,17 \mathrm{~mm}$ para a precipitação máxima diária anual. Adicionalmente, no geral, as superestimativas superaram as subestimativas, em termos absolutos. Destaca-se ainda que na literatura não são encontrados estudos de validação da estimativa de precipitação máxima diária anual do produto 3B42 do satélite TRMM.

Para o coeficiente de concordância, não foram encontradas boas exatidões entre os dados simulados e observados. $O$ posto com maior aproximação entre os dados foi o São José do Barreiro, com $59 \%$ de adequação. Vale ressaltar que os postos Fazenda das Garrafas e São José do Barreiro, estão contidos no mesmo pixel e por isso apresentaram a mesma média de precipitação estimada pelo satélite, contudo, a adequação entre os dados foram a menor e a maior encontrada, respectivamente. Assim, pode-se verificar que, para chuvas intensas e pontuais, a abrangência do pixel do satélite promove uma defasagem do acerto da lâmina precipitada. Além disso, Araújo et al. (2018) relatam que por possuir uma resolução temporal de 3 horas, o satélite TRMM pode não estimar precisamente eventos específicos de chuva, como os que ocasionam a máxima diária anual.

\section{CONCLUSÕES}

A validação das séries diárias de precipitação estimadas pelo produto 3B42 do satélite TRMM, para o período de 1998 a 2016 dos 6 postos pluviométricos da sub-bacia Paraíba do Sul, mostrou fraca correlação de Pearson $(0,29)$. Isto pode estar associado à área de abrangência do pixel, levando à diluição dos dados estimados em escala diária além do curto tempo de estimativa ( 3 horas) do sensor do satélite.

$\mathrm{Na}$ escala mensal, os coeficientes de determinação apresentaram boa adequação entre os dados, assim como a correlação de Pearson, que teve alto grau estatístico de dependência entre as variáveis. O mesmo aconteceu com 0 coeficiente de concordância (93\%), o que evidencia a compensação dos erros em escala temporal de acumulação pluvial. Portanto, a utilização do TRMM, em escala mensal, é a mais indicada.

A validação das séries de precipitação máxima diária anual não apresentou constância na correlação de Pearson e baixos valores para o grau de concordância entre os dados estimados e observados, o que denota que para eventos pluviais de alta intensidade e curta duração não é identificada precisão satisfatória.

\section{REFERÊNCIAS BIBLIOGRÁFICAS}

AGÊNCIA NACIONAL DE ÁGUAS (ANA). Séries históricas de estações. Disponível em: <http://www.snirh.gov.br/hidroweb/serieshistoricas>. Acesso em: 09 set. 2019. 
AGÊNCIA NACIONAL DE ÁGUAS (ANA). Diretrizes e análises recomendadas para a consistência de dados pluviométricos, ANA, 2012, 18p.

AGÊNCIA NACIONAL DE ÁGUAS (ANA). Plano de Ações Complementares para a Gestão da Crise Hídrica na Bacia do Rio Paraíba do Sul. ANA, 2015, 60p.

ALMEIDA, K. N. de et al. Performance analysis of TRMM satellite in precipitation estimation for the Itapemirim River basin, Espírito Santo state, Brazil. Theoretical and Applied Climatology, v. 141; p. 791-802, 2020.

ALVARES, C. A. et al. Köppen's climate classification map for Brazil. Meteorologische Zeitschrift, v. 22, p. 711-728, 2013.

ANJOS, R.; CANDEIAS, A.L.B.; NÓBREGA, R.S. Caracterização das chuvas através do satélite TRMM em Petrolândia-PE. Revista Brasileira de Climatologia, v.18, jan/jun., 2016.

ARAÚJO, D. C. dos S. et al. Validação da precipitação estimada pelos produtos TRMM $3 B 42$ e GPM IMERG em bacia experimental de Pernambuco. In: SIMPÓSIO DE RECURSOS HÍDRICOS DO NORDESTE, 14., 2018. Maceió. Anais... Maceió: Associação Brasileira de Recursos Hídricos, 2018. p. 1-9.

ARAÚJO, S.R.; SANCHES, F.O.; FERREIRA, R.V. Metodologia para validação de dados TRMM para Uberaba (MG). Entre Lugar, v. 8, p. 131-141, 2017.

CAMPAROTTO, L.B. et al. Validação de dados termopluviométricos obtidos via sensoriamento remoto para o Estado de São Paulo. Revista Brasileira de Engenharia Agrícola e Ambiental, v. 17, n. 6, p. 665-671, 2013.

CARVALHO, S. M. I. de; SHOEGIMA, T. F.; NERY, J. T. Análise quantitativa e espacial de distribuição dos períodos de chuva intensa e seca na Bacia Hidrográfica do rio Paraíba do Sul. In: CONGRESSO NACIONAL DE GEOGRAFIA FÍSICA. Anais... Campinas: Instituto de Geociências - Unicamp, 2017. p. 1-13.

COLLISCHONN, B. Uso de precipitação estimada pelo satélite TRMM em modelo hidrológico distribuído. Porto Alegre: Universidade Federal do Rio Grande do Sul. 2006. 128p. Dissertação de Mestrado.

COLLISCHONN, B.; ALLASIA, D.; COLLISCHONN, W.; TUCCI, C.E.M. Desempenho do satélite TRMM na estimativa de precipitação sobre a bacia do Paraguai superior. Revista Brasileira de Cartografia, Porto Alegre, v.59, n.1, p.93-99, 2007.

COLLISHONN, B.; COLLISHONN, W; TUCCI, C. Análise do campo de precipitação gerado pelo satélite TRMM sobre a bacia do São Francisco até Três Marias. In: Simpósio de Recursos Hídricos do Sul. Anais..., Curitiba, 2006.

DA SILVA, D.F.; PRELA-PANTANO, A.; CAMARGO, M.B.P. Análise de dados de precipitação estimados pelo satélite TRMM para o Vale do Médio ParapanemaSP. Revista Engenharia na agricultura - REVENG, Viçosa - MG, v.21 n.2, mar/abr. 2013.

DANCEY, C; REIDY, J. Estatística Sem Matemática para Psicologia: Usando SPSS para Windows. Artmed, 2006, 7 ed.

FENSTERSEIFER, C., ALLASIA, D.G., PAZ, A.R. Avaliação do produto de precipitação TRMM 3B42 no sul do Brasil. J. American Water Res. Association, v. 52, n. (2), p. 367-375, 2016. 
FERREIRA, S. P.; VARONE, F.; ALVES, R. de C. M. Uso do TRMM para a correção estatística da chuva prevista pelo modelo BRAMS. IV Simpósio Brasileiro de Ciências Geodésicas e Tecnologias de Geoinformação, p. 01-09, 2012.

FIGUEIREDO FILHO, D.B.; SILVA JÚNIOR, J.A. Desvendando os mistérios do coeficiente de correlação de Pearson (r). Revista Política Hoje, v. 18, n. 1, p. 115-46, 2009.

FIGUEIREDO, J.A.S.; DRUMM, E.; RODRIGUES, M.A.S.; SPILKI, F.R. The Rio dos Sinos watershed: an economic and social space and its interface with environmental status. Brazilian Journal of Biology, v. 70, p.1131-1136, 2010.

GARCIA, J.I.B.; PAIVA, E.M.C.D. de. Monitoramento hidrológico e modelagem da drenagem urbana da bacia do Arroio Cancela - RS. Revista Brasileira de Recursos Hídricos, v. 11, p. 1-10, 2006.

KARASEVA, M.; PRAKASH, S.; GAIROLA, R. Validation of high-resolution TRMM3B43 precipitation product using rain gauge measurements over Kyrgyzstan. Theoretical and Applied Climatology, vol. 108, n. 1-2, p. 147-157, 2011.

KIDDER, S. Q.; HAAR, V. Satellite Meteorology: an introduction. Academic Press, 1995.

KOTTEK, M. et al. World Map of the Köppen-Geiger climate classification updated. Meteorologische Zeitschrift, v. 15, p. 259-263, 2006.

KUMMEROW, C. et al. The Status of the Tropical Rainfall Measuring Mission (TRMM) after Two Years in Orbit. Journal of Applied Meteorology, v. 39, p. 1965-1982, 2000.

MACEDO, M. J. H. Aplicações de Redes Neurais Artificiais e Satélite TRMM na Modelagem Chuva-Vazão da Bacia Hidrográfica do Rio Paraguaçu/BA. Tese de Doutorado. Universidade Federal de Campina Grande, 2013.

MELLO, C. R. DE; SILVA, A. M. Hidrologia: Princípios e aplicações em sistemas agrícolas. Lavras: UFLA, 2013. 455p.

MELO NETO, J. O. Análise de Sensibilidade Escalar do Modelo Hidrológico SWAT. 2012. 149 p. Dissertação (Mestrado em Recursos Hídricos em Sistemas Agrícolas) - Universidade Federal de Lavras, Lavras, 2012.

MOREIRA, M. A. et al. Variação espectral e eficiência de uso da radiação fotossinteticamente ativa em ensaio com genótipos de trigo. Bragantia, v. 64, n. 3, p. 331-338, 2005.

NÓBREGA, R.S.; DE SOUZA, Ê.P.; GALVÍNCIO, J.D. Análise da Estimativa de Precipitação do TRMM na Sub-Bacia da Amazônia Ocidental. Revista de Geografia, v. 25, p. 06-20, 2008.

PEREIRA, G. et al. Avaliação dos Dados de Precipitação Estimados pelo Satélite TRMM para o Brasil. Revista Brasileira de Recursos Hídricos. Volume 18 n. 3, p. 139- 148, 2013.

PESSI, D.D. et al. Validação das estimativas de precipitação do satélite TRMM no Estado de Mato Grosso, Brasil. Revista de Ciências Agrárias, v. 42, n. 1, 2019. 
SILVA, D.F.; PANTANO, A.P.; CAMARGO, M.B.P. Análise de dados de precipitação estimados pelo satélite TRMM para o vale do médio Parapanema SP. Revista Engenharia na Agricultura, v. 21, p. 138-147, 2013.

SILVA, D.F.; PANTANO, A.P.; CAMARGO, M.B.P. Análise de dados de precipitação estimados pelo satélite TRMM para o vale do médio Parapanema SP. Revista Engenharia na Agricultura, v. 21, n.2, p. 138-147, 2013.

SOARES, A. S. D.; PAZ, A. R. da; PICCILI, D. G. A. Avaliação das estimativas de chuva do satélite TRMM no Estado da Paraíba. Revista Brasileira de Recursos Hídricos, v. 21, n.2, p. 288-299, 2016.

VIANA, D. R.; FERREIRA, N. J.; CONFORTE, J. C. Avaliação das estimativas de precipitação $3 B 42$ e 3B43 do satélite TRMM na Região Sul do Brasil. In: CONGRESSO BRASILEIRO DE METEOROLOGIA, 16., 2010. Belém. Anais... São José dos Campos: INPE, 2010, CD-ROM.

WILLMOTT, C.J.; ACKLESON, S.G.; DAVIS, J.J. Statistics for the evaluation and comparison of models. Journal of Geography Research, v.90, n.5, p.8995-9005, 1985 